\title{
Generation of Ion Beams from High- $Z$ Target Irradiated by Laser Pulse of Ultra-Relativistic Intensity
}

\author{
J. DOMAŃSKI* AND J. BADZIAK \\ Institute of Plasma Physics and Laser Microfusion, Warsaw, Poland \\ Doi: $10.12693 /$ APhysPolA.138.586 \\ *e-mail: jaroslaw.domanski@ifpilm.pl
}

\begin{abstract}
High-energy heavy ion beams produced by conventional RF-driven accelerators are a basic research tool in nuclear and particle physics but they also find applications in other domains such as high energy-density physics or materials science. With their potential to be more compact and less complex, laser-driven ion accelerators are considered to be a promising alternative or supplement to RF-driven accelerators. However, for achieving $\mathrm{GeV}$ or multi-GeV ion energies, multi-PW laser drivers and ultrarelativistic laser beam intensities $\left(\geq 10^{23} \mathrm{~W} / \mathrm{cm}^{2}\right)$ are required. Such multi-PW lasers are currently being built, in particular in Europe, as part of the Extreme Light Infrastructure project. In this paper, the results of numerical studies on the acceleration of heavy ions from a gold sub-micrometer target irradiated by a $30-\mathrm{fs}$, multi-PW laser pulse of ultra-relativistic intensity $\left(\approx 10^{23} \mathrm{~W} / \mathrm{cm}^{2}\right)$ and with linear or circular polarization are reported. The numerical simulations were performed using advanced, multi-dimensional (2D3V) particle-in-cell code PICDOM which includes, in particular, the dynamic ionization of the target and the accelerated ions as well as radiation losses due to synchrotron radiation emitted by ultra-relativistic electrons. It was found that the effect of radiation losses on the ion beam parameters is stronger for the linear polarization laser beam and radiation losses influences first of all the maximum ion energy. However, the effect of radiation losses on the mean ion energy, ion beam fluence and intensity and the ion pulse shape is insignificant, below $1-2 \%$, both for the circular or linear polarization beam. For both beam polarizations, the multi-PW laser pulse generates an ultra-intense multi-GeV gold ion beam of intensities $\left(>10^{20} \mathrm{~W} / \mathrm{cm}^{2}\right)$ and durations $(<100 \mathrm{fs})$ which are presently unattainable in $\mathrm{RF}$-driven accelerators.
\end{abstract}

topics: heavy ion beam, laser-driven ion acceleration, laser-plasma interaction, multi-PW laser

\section{Introduction}

High energy heavy ion beams are a basic research tool in nuclear and particle physics. They also find applications in other domains, such as high energy density physics or materials science. Such ion beams are usually produced by conventional radio frequency $(\mathrm{RF})$-driven accelerators but can also be generated by laser-driven accelerators [1-3]. RF-driven accelerators can generate ion beams with high $(\sim \mathrm{GeV})$ and ultra-high $(\sim \mathrm{TeV})$ energies and extremely narrow energy spectra; however, they are quite complex and large devices. Laser-driven accelerators are much more compact facilities and are able to produce short (picosecond) ion beams of high intensity/current density, unattainable in conventional $\mathrm{RF}$ accelerators. Unfortunately, the energy spectra of ion beams produced by such accelerators are typically wide. Moreover, to produce in these accelerators ion beams with the $\mathrm{GeV}$ or multi-GeV ion energies desired in numerous applications [1-5], multi-PW short-pulse laser drivers and ultra-relativistic laser intensities $\left(\approx 10^{23} \mathrm{~W} / \mathrm{cm}^{2}\right.$ or higher) are required [6-12].
Laser facilities of such parameters are currently being built as part of the pan-European Extreme Light Infrastructure (ELI) project [5]. In March 2019, the ELI-Nuclear Physics laboratory launched a $10 \mathrm{PW}$ laser, the most powerful laser ever built in the world, and preliminary experiments on laserdriven ion acceleration with the use of this laser will be started soon.

At present, the acceleration of heavy ions at ultra-relativistic laser intensities is a poorly explored research field [11-15] and a thorough understanding of the acceleration process and properties of the ion beams produced requires extensive and detailed studies, both experimental and numerical ones. This paper presents the results of a numerical investigation of the acceleration of heavy $(\mathrm{Au})$ ions from a thin $100 \mathrm{~nm}$ gold target irradiated by a circularly (CP) or linearly (LP)polarized 30 fs multi-PW laser pulse with ultrarelativistic intensity of $10^{23} \mathrm{~W} / \mathrm{cm}^{2}$ and the focal spot diameter equal to $3 \mu \mathrm{m}$. The parameters of the laser pulse correspond to the predicted parameters of the ELI-NP laser [5, 16]. The density of the target corresponded to the density of 
the solid state, set at $5.9 \times 10^{22}$ atoms $/ \mathrm{cm}^{3}$. A preplasma layer of the shape described by the exponential function and thickness equal to $20 \mathrm{~nm}$ was placed at the front of the target. It is assumed that the contrast ratio of the laser pulse is high enough not to destroy the target by the laser pre-pulse. This assumption is based on experiments, such as the one presented in [17], where targets of similar or even smaller thicknesses were used. The simulations were performed using multidimensional (2D3V) particle-in-cell PICDOM code which includes the ionisation process of gold atoms and radiation losses due to synchrotron radiation emitted by ultra-relativistic electrons. In the second section of this paper, the structure of the PICDOM code is described while the third section presents the results of the performed simulations and their discussion.

\section{The PICDOM code}

In the particle-in-cell codes, the plasma is described by the electrons and ions moving in the electromagnetic field generated by their own charges and the laser beam. The amount of real particles in the system is typically huge. For this reason, the amount of particles in the simulations is reduced and each macroparticle (present in simulations) represents many real particles.

In the PICDOM code, similarly to other PIC codes, the electromagnetic fields are calculated on a network of discrete Eulerian points (forming cells). Based on Maxwell's equations and assuming that the calculating space overlaps with the $x-y$ surface, we can write equations for the perpendicular $z$-component of electric and magnetic fields

$$
\begin{aligned}
& \frac{\partial^{2} E_{z}}{\partial t^{2}}=c^{2}\left(\frac{\partial^{2} E_{z}}{\partial x^{2}}+\frac{\partial^{2} E_{z}}{\partial y^{2}}\right)-\frac{1}{\varepsilon_{0}} \frac{\partial j_{z}}{\partial t}, \\
& \frac{\partial^{2} B_{z}}{\partial t^{2}}=c^{2}\left(\frac{\partial^{2} B_{z}}{\partial x^{2}}+\frac{\partial^{2} B_{z}}{\partial y^{2}}\right)+\frac{1}{\varepsilon_{0}}\left(\frac{\partial j_{y}}{\partial x}+\frac{\partial j_{x}}{\partial y}\right),
\end{aligned}
$$

where $j_{x}, j_{y}$, and $j_{z}$ are the components of the current. The $x$ and $y$ components of the electric and magnetic field are connected with the $z$ component in the following way:

$$
\begin{aligned}
& \frac{\partial E_{x}}{\partial t}=c^{2} \frac{\partial B_{z}}{\partial y}-\frac{1}{\varepsilon_{0}} j_{x}, \quad \frac{\partial B_{x}}{\partial t}=-\frac{\partial E_{z}}{\partial y}, \\
& \frac{\partial E_{y}}{\partial t}=-c^{2} \frac{\partial B_{z}}{\partial x}-\frac{1}{\varepsilon_{0}} j_{y}, \quad \frac{\partial B_{y}}{\partial t}=\frac{\partial E_{z}}{\partial x} .
\end{aligned}
$$

After discretisation, these equations were implemented in the code.

Macroparticles are tracked in a continuous $x-y$ space. Despite the fact that the macroparticles can move in the 2D space, all three components of their velocities are calculated. The motion of the ion macroparticles in the electromagnetic field is described by

$$
\frac{\mathrm{d} \boldsymbol{p}_{i}}{\mathrm{~d} t}=\boldsymbol{f}_{\mathrm{L}}=q(\boldsymbol{E}+\boldsymbol{v} \times \boldsymbol{B}),
$$

where $\boldsymbol{p}_{i}$ is the particle momentum and $\boldsymbol{f}_{\mathrm{L}}$ is the Lorentz force. The local electric $\boldsymbol{E}$ and magnetic $\boldsymbol{B}$ fields interacting with macroparticles are calculated as the weighted average from the value corresponding to the cells occupied by the particles. To take into account the radiation losses (the radiation reaction force) in case of electron macroparticles, the Sokolov model is used [18, 19]:

$$
\frac{\partial \boldsymbol{p}_{e}}{\partial t}=\boldsymbol{f}_{\mathrm{L}}-e c\left(\boldsymbol{\delta} \boldsymbol{\beta}_{e} \times \boldsymbol{B}\right)-\gamma^{2}\left(\boldsymbol{f}_{\mathrm{L}} \cdot \boldsymbol{\delta} \boldsymbol{\beta}_{e}\right) \boldsymbol{\beta}_{e},
$$

where $\gamma$ is the relativistic factor, $\boldsymbol{\beta}_{e}=\boldsymbol{v} / c$ is the dimensionless particle velocity and $\delta \boldsymbol{\beta}_{e}$ is defined accordingly:

$$
\delta \boldsymbol{\beta}_{e}=\frac{\tau_{r}}{m_{e} c} \frac{\boldsymbol{f}_{\mathrm{L}}-\boldsymbol{\beta}_{e}\left(\boldsymbol{\beta}_{e} \cdot \boldsymbol{f}_{\mathrm{L}}\right)}{1+\frac{\tau_{r}}{m_{e} c}\left(\boldsymbol{\beta}_{e} \cdot \boldsymbol{f}_{\mathrm{L}}\right)},
$$

where $\tau_{r}=e^{2} /\left(6 \pi \varepsilon_{0} m_{e} c^{3}\right)$ is the characteristic time of radiation emission, $m_{e}$ is the electron mass and $e$ is the elementary charge. The implementation of the equation of motion is done with the help of the two-step rotation method presented by Bridsall and Langdon [20], expanded by a special correction for radiation losses.

The interaction between particles and electromagnetic fields may result in the ionisation effect. This effect is described using the AmmosovDelone-Krainov formula $[14,21]$ :

$W=\frac{\omega_{\alpha}}{8 \pi} \frac{E}{E_{\mathrm{H}}}\left(\frac{4 \pi E_{\mathrm{H}} \zeta^{3}}{E n^{* 4}}\right)^{2 n^{*}} \exp \left(-\frac{2}{3} \frac{E_{\mathrm{H}}}{E} \frac{\zeta^{3}}{n^{* 3}}\right)$,

where $\omega_{\alpha}=4.13 \times 10^{-16} \mathrm{~s}^{-1}$ is the atomic unit frequency, $U_{\mathrm{H}}=1312 \mathrm{~kJ} / \mathrm{mol}$ is the ionisation potential of a hydrogen atom, $E_{H}=5.14 \times 10^{11} \mathrm{~V} / \mathrm{m}$ is the corresponding electric field, $n^{*}=\zeta \sqrt{U_{\mathrm{H}} / U_{i}}$ is the effective principal quantum number, $U_{i}$ is the ionization potential, $\zeta$ is the final charge state, and $E$ is the local electric field. Then, the probability of ionisation is expressed by

$$
P=1-\mathrm{e}^{-W \Delta t} \text {. }
$$

Details of the implementation and incorporation of (7) in the code are described in [22]. This formula is correct when the Keldysh parameter is smaller than one and ionisation energy is smaller than the electron rest energy. These conditions are met for the investigated range of the laser pulse intensity [23, 24].

The last step necessary to close the loop is to calculate the current density used to establish the new value of the electric and magnetic field. For this purpose, the modified version of the procedure proposed by Villasenor and Buneman was used [25]. In our code, the charge flows through the boundaries of additional shifted network cells are calculated. Owing to that, the current component vectors are parallel to the boundaries of the cells of the original network. Additionally, the procedure for the current density $z$-component calculation was added. These changes enabled us to calculate all electric and magnetic field vectors in one point in the centre of the cell. 


\section{Results and discussion}

The 2D spatial distributions of ion density $(\mathrm{a}, \mathrm{d})$, electron density $(b, e)$ and ionisation level $(c, f)$ for linear (LP, a, b, c) and circular (CP, d, e, f) polarisation of the laser pulse at the early $t=30 \mathrm{fs}$ and late $t=100 \mathrm{fs}$ stage of acceleration are shown in Figs. 1 and 2 , respectively. The results are quite similar for two types of investigated polarisation, both at the early and late stage of acceleration. The biggest difference is a higher amount of hot electrons moving far away from the ions for LP in the early stage of acceleration (Fig. 1b). It is worth noting that for all cases the majority of the electrons co-moves with the ions and the ions of the highest ionisation level are situated in the centre of the target, the region of direct laser interaction. During the interaction of an ultra-intense laser beam with a solid target, the target atoms are ionised by the strong electric field of the laser beam. After the plasma has been produced, the created ions are additionally ionised by the strong electric field generated in the plasma as a result of the local imbalance between the positive charge of ions and the negative charge of electrons surrounding the ions. These fields are strongest in the centre, where the amount of hot electrons is the highest. For this reason, the highest ionisation level in the centre of the target is fully understandable.

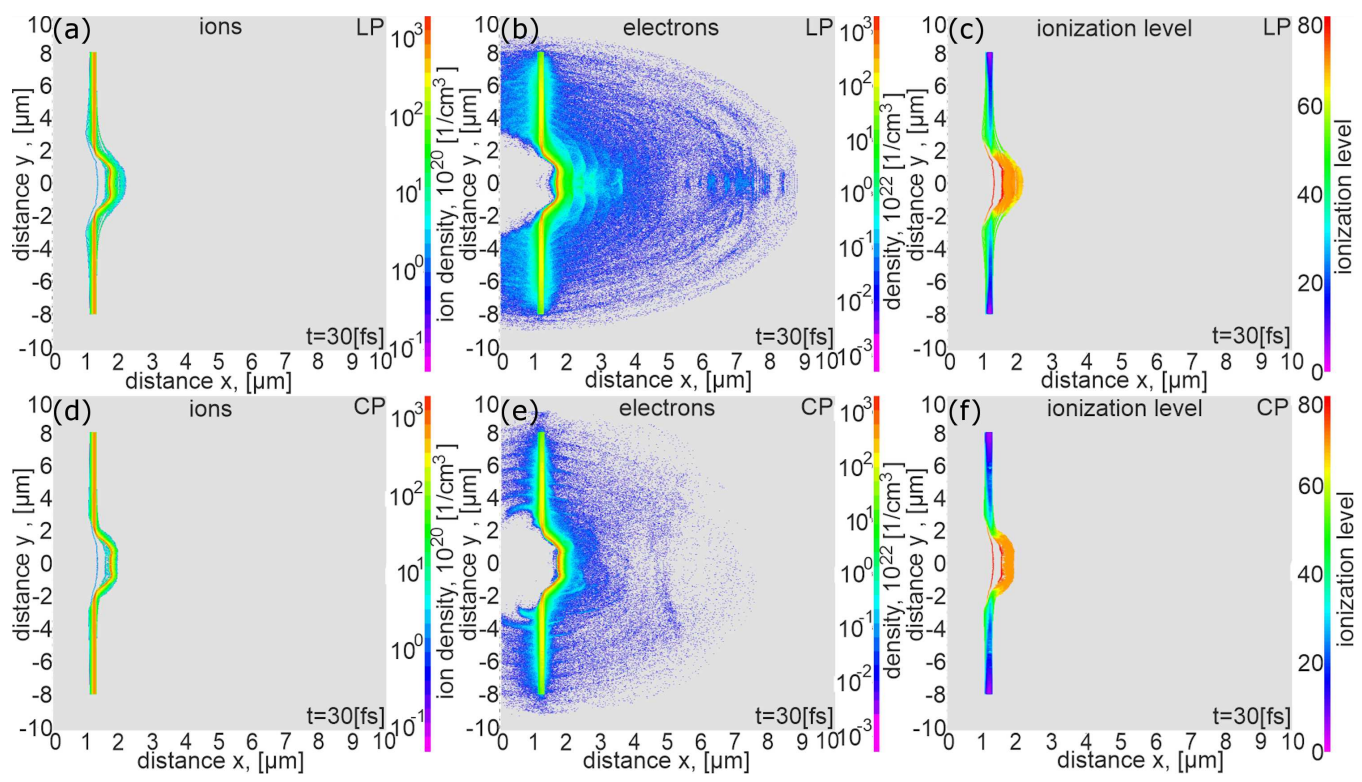

Fig. 1. The 2D spatial distributions of ion density (a, d), electron density (b, e) and ionisation level (c, f) for linear (LP, a, b, c) and circular (CP, d, e, f) laser polarisation at the early ( $t=30 \mathrm{fs})$ stage of ion acceleration.

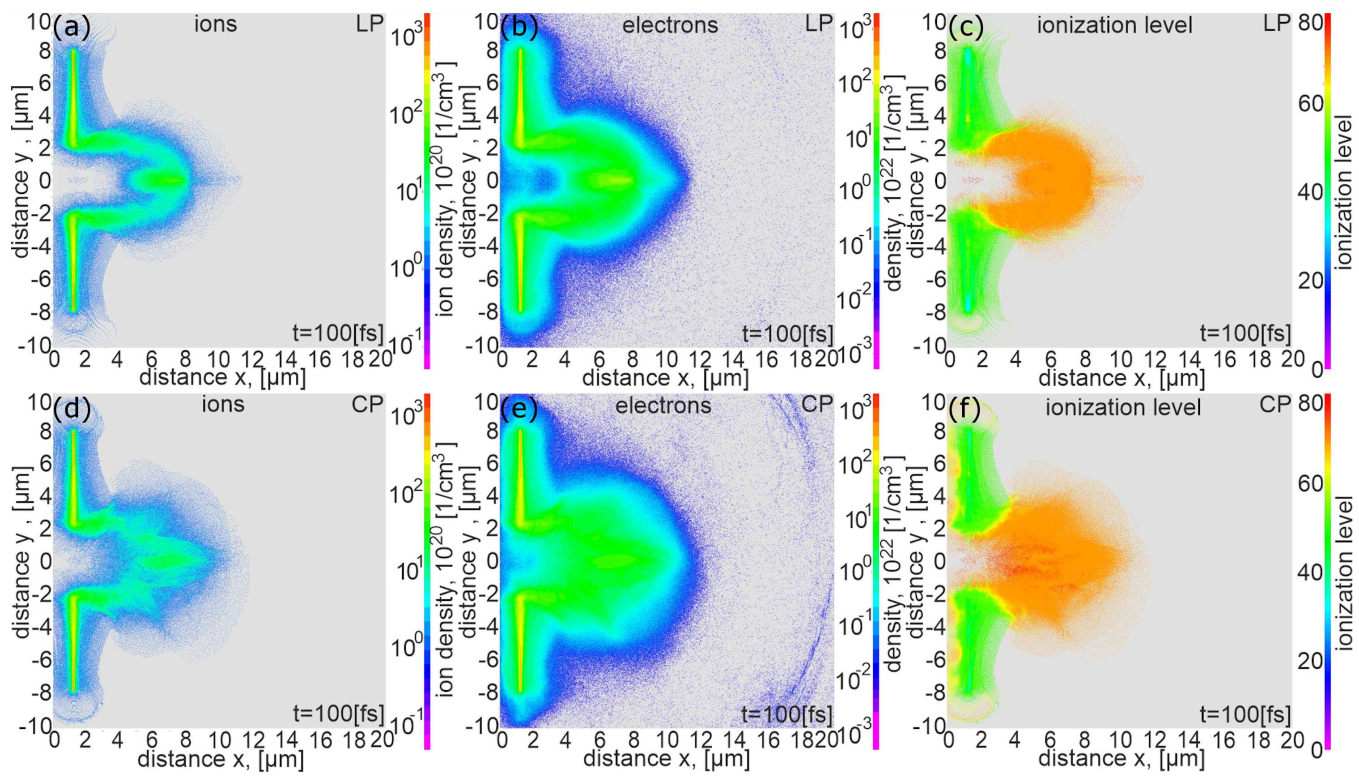

Fig. 2. The 2D spatial distributions of ion density (a, d), electron density (b, e) and ionisation level (c, f) for linear (LP, a, b , c) and circular $(\mathrm{CP}, \mathrm{d}, \mathrm{e}, \mathrm{f})$ polarisation at the late $(t=100 \mathrm{fs})$ stage of ion acceleration. 

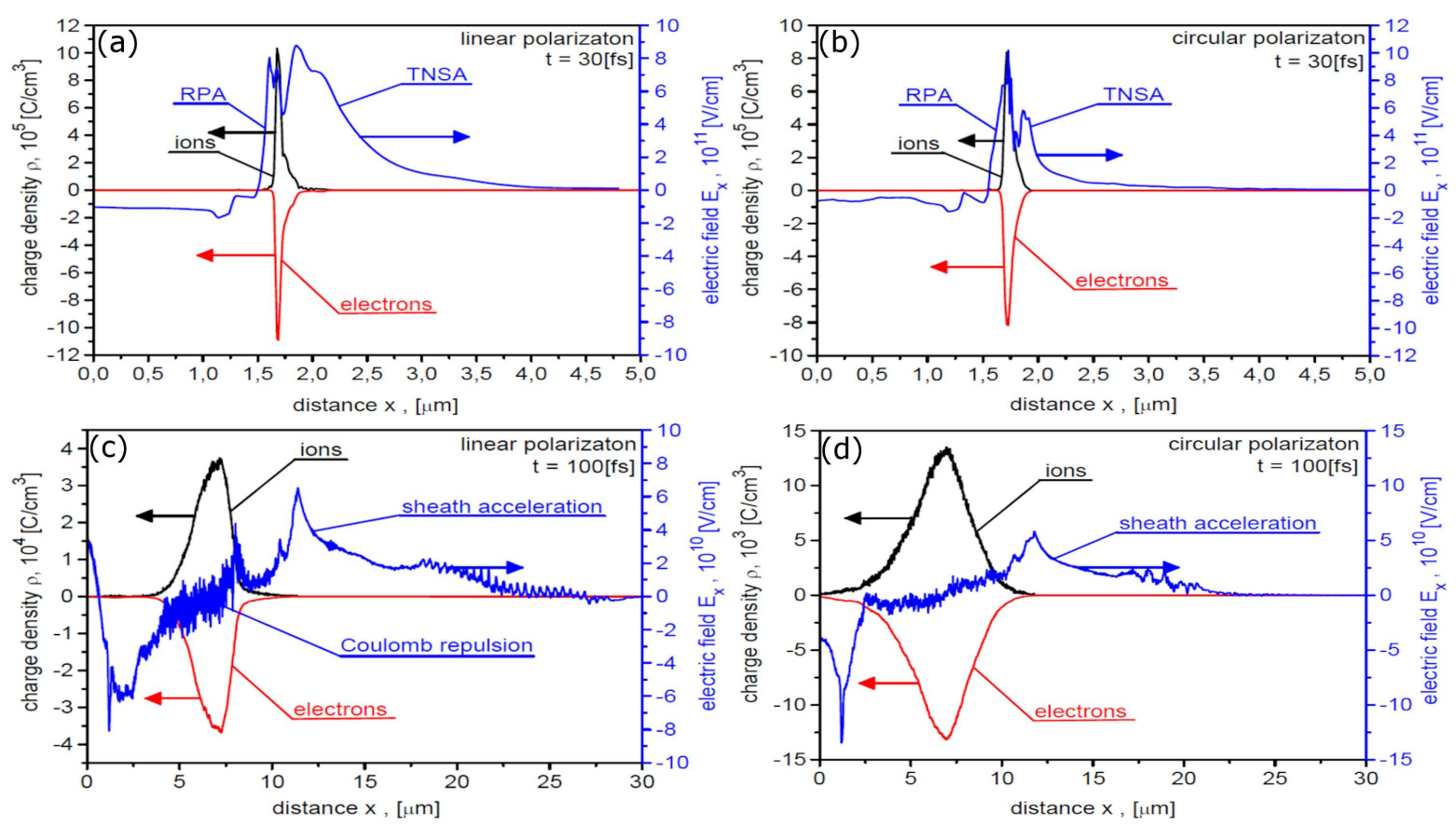

Fig. 3. The spatial profiles of the ion density, the electron density and the electric field accelerating ions $E_{x}$, along the laser beam axis, for linear and circular polarisation at the two stages of ion acceleration.

In order to better understand the ion acceleration process, the spatial profiles of the ion density, electron density and electric field accelerating ions $E_{x}$, along the laser beam axis, for linear and circular polarisation at two stages of ion acceleration are presented (Fig. 3). At the early stage of acceleration, during direct interaction of the laser pulse with the target, it is visible that ions from the front side of the target are accelerated by the laser pulse radiation pressure (the radiation pressure acceleration (RPA) mechanism $[1-3,26])$ while the ions from the rear side are accelerated by the target normal sheath acceleration (TNSA) mechanism [1-3, 27] connected with the electric field generated by hot electrons. A significant difference in the strength of the TNSA field for two types of the investigated polarisation is visible. This difference is connected with the amount of hot electrons. At the late stage of acceleration, after the end of direct interaction between the laser pulse and the target, the accelerating fields are much weaker but the sheath acceleration of the ions from the rear side of the beam is still noticeable. The efficiency of this acceleration is quite similar for $\mathrm{CP}$ and LP.

In Fig. 4, the ionisation spectra of ions produced from the gold target irradiated by a linearly and circularly polarised laser pulse at the late stage of acceleration are presented. Only small differences between the two types of laser polarisation are visible. The peaks visible on the spectra correspond to gold ionisation energy gaps [23]. It is worth noting that the $\mathrm{Au}^{69+}$ ions dominate in the centre of the target (see Fig. 1c,f and Fig. 2c,f) and the majority of the ion beam energy is stored in the $\mathrm{Au}^{69+}$ ions.

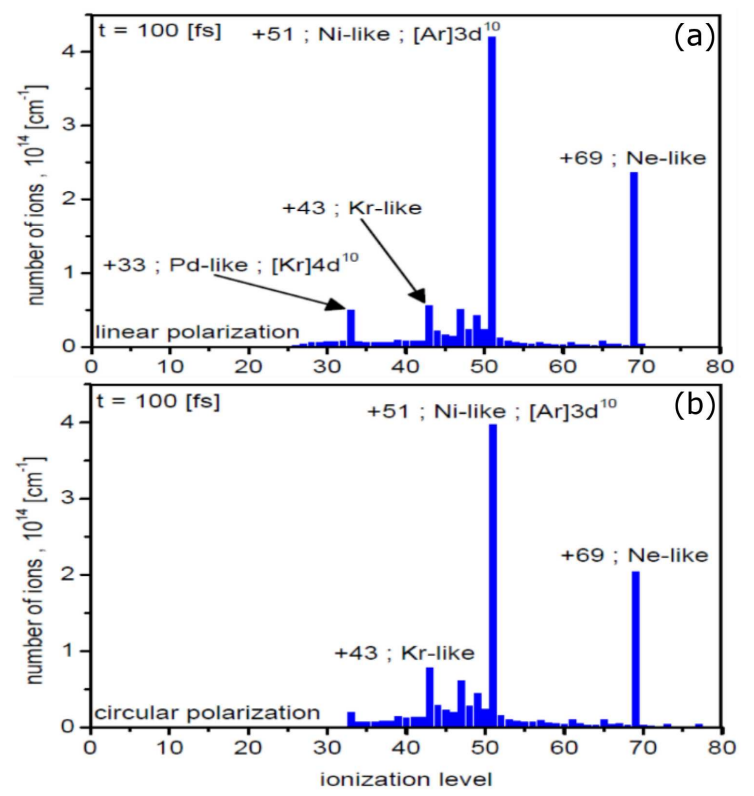

Fig. 4. The ionisation spectra of ions produced from the gold target irradiated by a linearly or circularly polarised laser pulse at the late stage of ion acceleration.

The ion energy spectra for linear and circular polarisation of the laser pulse at the end of the acceleration process are presented in Fig. 5. Both for LP and CP, the energy spectra are wide and quasi-Maxwellian. The main differences between the spectra are observed in the long high energy tail. The maximum energy of ions is higher for the circular polarisation and reaches around $40 \mathrm{GeV}$. 


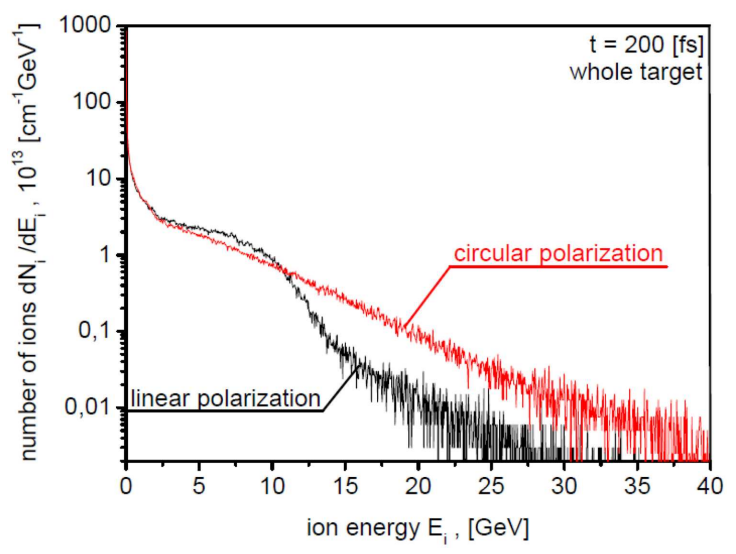

Fig. 5. The ion energy spectra for linear and circular polarisation of the laser pulse at the end of the acceleration process.

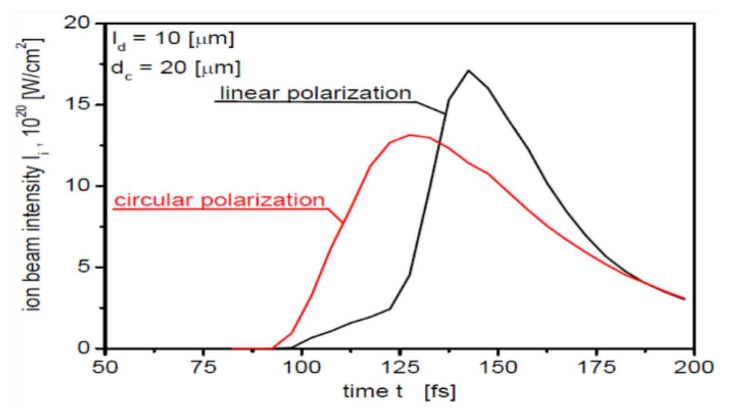

Fig. 6. Temporal distributions of ion beam intensity recorded $10 \mu \mathrm{m}$ behind the target and averaged over the $y$ range equal to $20 \mu \mathrm{m}$.

It is worth to mention that the energy conversion from the laser beam to the accelerated ions is equal to $23.2 \%$ for CP and $20.7 \%$ for LP.

Important parameters of ultra-intense ion beams are the beam peak intensity $I_{i}$, ion pulse duration $\tau_{i}$ and shape as well as the collimation of the beam. In Fig. 6, the temporal distributions of ion beam intensity, recorded $10 \mu \mathrm{m}$ behind the target and averaged over the $y$ range equal to $20 \mu \mathrm{m}$, are presented. For linear polarisation, shorter and more intense ion beams are observed, despite the fact that the highest energy of ions is observed for CP. It is particularly interesting that for both beam polarisations, the multi-PW laser pulse generates ultra-intense multi-GeV gold ion beams of intensities $\left(>10^{21} \mathrm{~W} / \mathrm{cm}^{2}\right)$ and durations $(<100 \mathrm{fs})$ which are presently unattainable in RF-driven accelerators. The ion pulse is about twice as long as the laser pulse due to the ion velocity dispersion. Additionally, as a result of the action of radial forces accelerating ions, the ion beam is much wider than the laser beam.

In Fig. 7, the angular distributions of ion beam intensity for linear (a) and circular (b) polarisation of laser pulse and three time steps, recorded $10 \mu \mathrm{m}$ behind the target, are presented. Lower angular divergences are observed for the ion beam produced

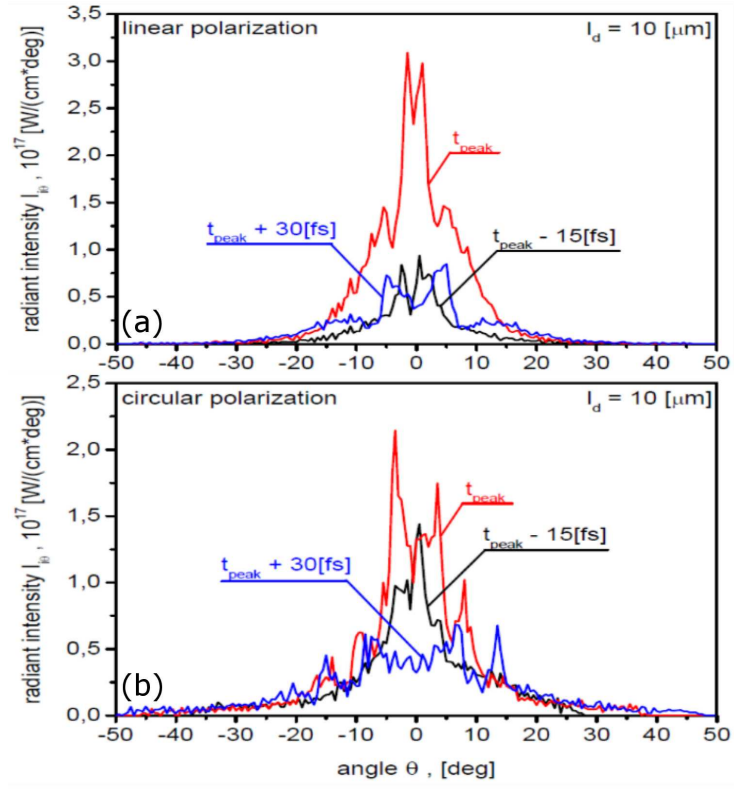

Fig. 7. Angular distributions of ion beam intensity for linear (a) and circular (b) polarisation of laser pulse recorded $10 \mu \mathrm{m}$ behind the target at three different times.

by the linearly polarised laser pulse. To conclude, for the linear polarisation of the laser, shorter and more intense ion beams of the lower angular divergence are produced. However, the differences in the parameters of the ion beam for linear and circular polarization are not so pronounced.

During the process of ion acceleration at ultrarelativistic laser intensity, ultra-relativistic electrons with the Lorenz factor $\gamma \gg 1$ can be produced. Such electrons can lose part of their energy due to synchrotron radiation. These radiation losses $(\mathrm{RL})$ can influence the ion acceleration process $[6,8]$. It was shown that RL can significantly influence the acceleration process of protons and deuterons by the ultra-relativistic laser [8]. For this reason, it is also important to study the influence of RL on the process of heavy ion acceleration. The radiation power density for linear $(a, b, c)$ and circular $(d, e, f)$ polarisation at three stages of acceleration: $t=20 \mathrm{fs}$ (a, d), $t=40 \mathrm{fs}$ (b, e) and $t=70 \mathrm{fs}(\mathrm{c}, \mathrm{f})$ is presented in Fig. 8. Radiation losses reach the maximum close to the end of direct laser target interactions and then start to dramatically decrease with time both for linear and circular polarisation of the laser pulse. Furthermore, the highest energy losses due to synchrotron radiation occur on the front of the ion beam, in the area of direct interaction between the laser and plasma.

The temporal distribution of the total radiation power for both types of laser polarisation is presented in Fig. 9. During direct interaction between the laser and target, the radiation losses are much higher for the LP laser beam. The radiation pressure acting on the target changes without oscillations for $\mathrm{CP}$ while it oscillates between zero and 


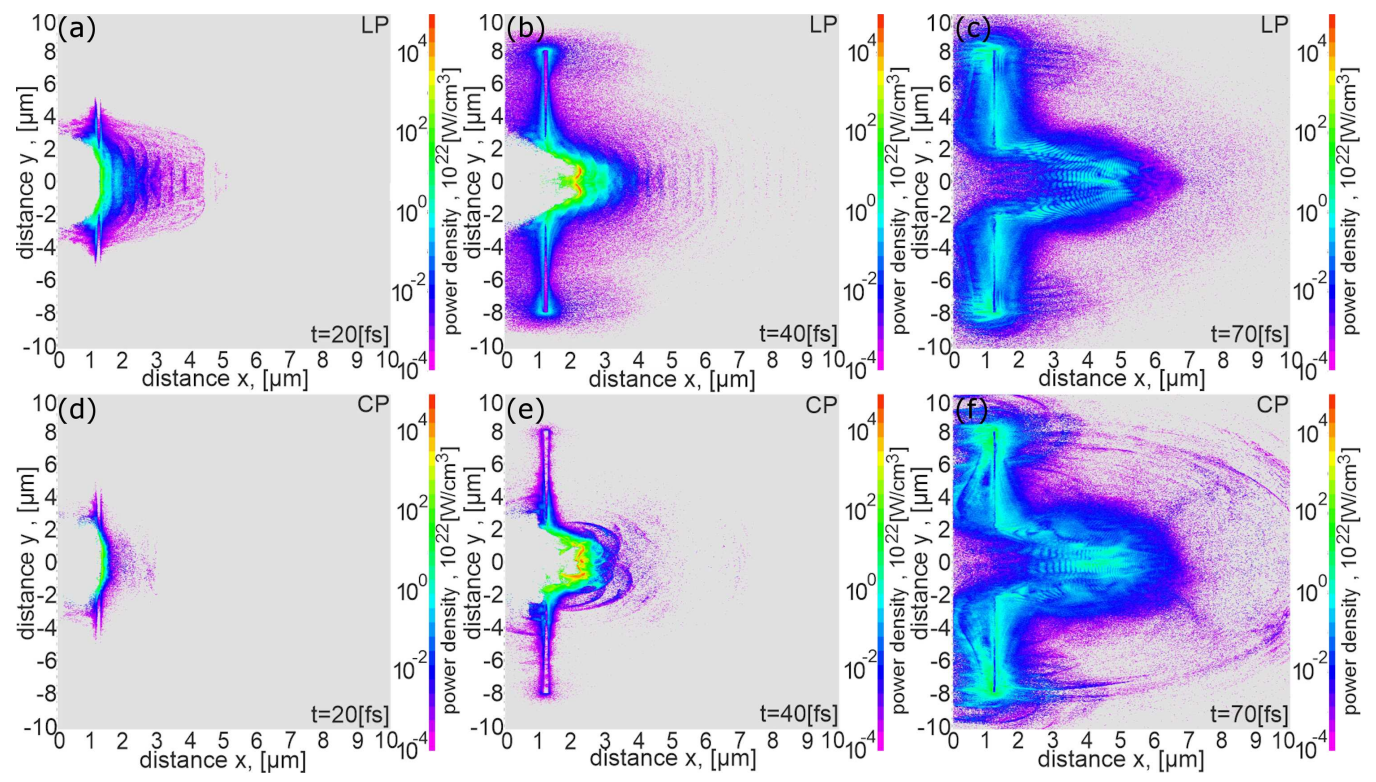

Fig. 8. The radiation power density for linear (a, b, c) and circular (d, e, f) laser polarisation at the three stages of acceleration: $t=20 \mathrm{fs}(\mathrm{a}, \mathrm{d}), t=40 \mathrm{fs}(\mathrm{b}, \mathrm{e})$ and $t=70 \mathrm{fs}(\mathrm{c}, \mathrm{f})$.

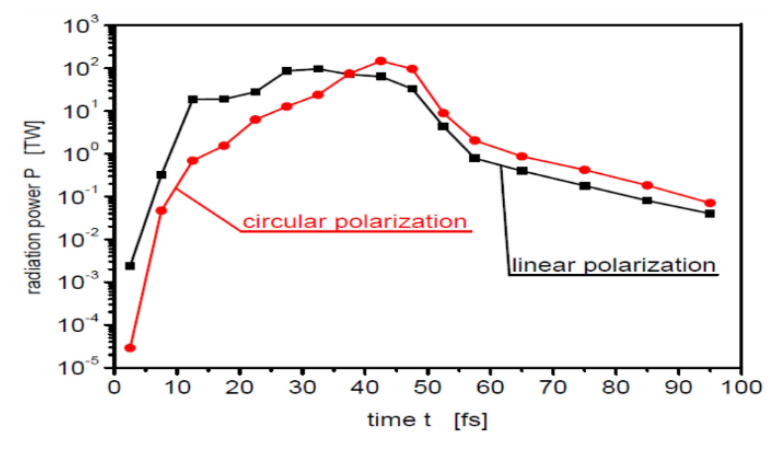

Fig. 9. Temporal distribution of the total radiation power for both types of laser polarisation.

TABLE I

The mean and maximum ion energies with and without radiation losses for two types of laser polarisations.

\begin{tabular}{l|l|c|c}
\hline \hline \multicolumn{2}{|c|}{} & With RL & Without RL \\
\hline \multirow{2}{*}{$\begin{array}{l}\text { linear } \\
\text { polarization }\end{array}$} & mean energy $[\mathrm{GeV}]$ & 1.645 & 1.664 \\
\cline { 2 - 4 } $\begin{array}{l}\text { circular } \\
\text { polarization }\end{array}$ & max. energy $[\mathrm{GeV}]$ & 35.137 & 39.798 \\
\cline { 2 - 4 } & max. energy $[\mathrm{GeV}]$ & 41.59 & 1.823 \\
\cline { 2 - 4 } & & & 43.564
\end{tabular}

the maximum value for LP. This effect leads to a difference in RL. During the late stage of acceleration, the RL are higher for CP which is caused by a higher amount of high energy electrons for circular polarisation. Despite this, finally it was found that the effect of RL on the ion beam parameter is stronger for the LP laser beams. However, the amount of energy transferred to the synchrotron radiation is lower than $1 \%$ of the total laser pulse energy and equal to $0.786 \%$ for LP and $0.702 \%$ for CP. To define the influence of RL on the parameters of the produced ion beams, additional simulations in which the radiation losses were not taken into account were made. The mean and maximum ion energies with and without the inclusion of the radiation losses for two types of laser polarizations are presented in Table I. The effect of RL is noticeable only for the maximum ion energies. The influence of RL on the mean ion energy, ion beam fluence and intensity as well as on the ion pulse shape is small and does not exceed 1-2\% both for LP and CP.

\section{Conclusions}

The process of heavy (gold) ion acceleration by a multi-PW laser pulse of ultra-relativistic intensity $\approx 10^{23} \mathrm{~W} / \mathrm{cm}^{2}$ to be available at the ELI infrastructure has been studied using a 2D3V PIC code. The hybrid ion acceleration process composed of the RPA stage followed by the sheath acceleration stage, driven by the electric field created by fast electrons moving far away from the ions, was demonstrated and discussed. The influence of the laser pulse polarisation on the ion acceleration process and parameters of the heavy ion beam produced by an ultra-relativistic laser pulse was shown. It was found that both the mean and maximum ion energies are higher for the circular polarisation (equal to $1.8 \mathrm{GeV}$ and $42 \mathrm{GeV}$, respectively) while shorter and more intense ion beam of lower angular divergence is produced by the linearly polarised laser pulse. However, the differences in the ion beam parameters for linear and circular polarisation are not very large. For both polarisations, the multi-PW laser pulse generates an ultra-intense multi-GeV gold ion beam of intensities $\left(>10^{21} \mathrm{~W} / \mathrm{cm}^{2}\right)$ and durations ( $\left.<100 \mathrm{fs}\right)$ which are presently unattainable in RF-driven accelerators. Finally, it was found that the influence of radiation losses due to synchrotron radiation on 
the process of ion acceleration is stronger for the linear polarisation of laser pulse and RL influences primarily the maximum ion energies. The effect of RL on the mean ion energy, ion beam fluence, intensity and ion pulse shape is small and does not exceed $1-2 \%$. Both for CP and LP, the amount of energy transferred to the synchrotron radiation is lower than $1 \%$ of the total laser pulse energy.

\section{Acknowledgments}

This work was supported in part by the EUROfusion Consortium (the RoHGIFE project) and has received partial funding from the Euratom research and training programme 2019-2020 under grant agreement No. 633053. The simulations were carried out with the support of the Interdisciplinary Center for Mathematical and Computational Modelling (ICM), University of Warsaw, under grant no. G57-20.

\section{References}

[1] H. Daido, M. Nishiuchi, A.S. Pirozhkov, Rep. Prog. Phys. 75, 056401 (2012).

[2] A. Macchi, M. Borghesi, M. Passoni, Rev. Mod. Phys. 85, 751 (2013).

[3] J. Badziak, J. Phys. Conf. Series 959, 012001 (2018).

[4] K.W.D. Ledingham, W. Galster, New J. Phys. 12, 045005 (2010).

[5] F. Negoita, M. Roth, P.G. Thirolf et al., Roman. Rep. Phys. 68, S37 (2016).

[6] M. Tamburini, F. Pegoraro, A. Di Piazza, C.N. Keitel, A. Macchi, New. J. Phys. 12, 123005 (2010).

[7] F.L. Zheng, H.Y. Wang, X.Q. Yan, T. Tajina, M.Y. Yu, X.T. He, Phys. Plasmas 19, 023111 (2012).

[8] R. Capdessus, P. McKenna, Phys. Rev. E 91, 053105 (2015).

[9] S.S. Bulanov, E. Esarey, C.B. Schroeder, S.V. Bulanov, T.Zh. Esirkepov, M. Kondo, W.P. Leemans, Phys. Plasmas 23, 056703 (2016).

[10] Y. Xu, J. Wang, X. Qi, M. Li, Y. Xing, L. Yang, W. Zhu, Phys. Plasmas 24, 033108 (2017).
[11] J. Domański, J. Badziak, Phys. Lett. A 382, 3412 (2018).

[12] J. Domański, J. Badziak, M. Marchwiany, Laser Part. Beams 36, 507 (2018).

[13] Extreme Light Infrastructure Project.

[14] D. Wu, B. Qiao, C. McGuffey, X.T. He, F.N. Beg, Phys. Plasmas 21, 123118 (2014).

[15] Jun Li, A.V. Arefiev, S.S. Bulanov, D. Kawahito, M. Bailly-Grandvaux, G.M. Petrov, C. McGuffey, F.N. Beg, Sci. Rep. 9, 666 (2019).

[16] D. Ursescu, G. Cheriaux, P. Audebert et al., Roman. Rep. Phys. 68, S11 (2016).

[17] J. Li, P. Forestier-Colleoni, M. BaillyGrandvaux et al., New J. Phys. 21, 103005 (2019).

[18] I.V. Sokolov, J.A. Nees, V.P. Yanovsky, N.M. Naumova, G.A. Mourou, Phys. Rev. E 81, 036412 (2010).

[19] R. Capdessusa, E. d'Humières, V. Tikhonchuk, EPJ Web Conf. 59, 17019 (2013).

[20] C.K. Bridsall, A.B. Langdon, Plasma Physics via Computer Simulation, McGraw Hill, New Jork 1985.

[21] M.V. Ammosov, N.B. Delone, V.P. Krainov, J. Exp. Theor. Phys. 64, 1191 (1986).

[22] M. Chen, E. Cormier-Michel, C.G.R. Geddes, D.L. Bruhwiler, L.L. Yu, E. Esarey, C.B. Schroeder, W.P. Leemans, J. Computat. Phys. 236, 220 (2013).

[23] A. Kramida, Yu. Ralchenko, J. Reader, and NIST ASD Team, NIST Atomic Spectra Database (ver. 5.5.2), National Institute of Standards and Technology, Gaithersburg (MD) 2018.

[24] V.S. Popov, Phys. Usp. 47, 855 (2004).

[25] J. Villasenor, O. Buneman, Comput. Phys. Commun. 69, 306 (1992).

[26] A. Macchi, F. Cattani, T.V. Liseykina, F. Cornalti, Phys. Rev. Lett. 94, 165003 (2005).

[27] S.C. Wilks, A.B. Langdon, T.E. Cowan et al., Phys. Plasmas 8, 542 (2001). 